\title{
Fuzzy Logic based Image De-noising and Enhancement for Grayscale Images
}

\author{
Suraj Kamya \\ Department of Electronics \& Communication \\ Lovely Professional University, Punjab
}

\author{
Madhuri Sachdeva \\ Department of Electronics \& Communication \\ Lovely Professional University, Punjab
}

\begin{abstract}
A picture is worth a thousand words. Trillions of digital images are used for different purposes in real life every day. Noise can corrupt the images in different ways which results in loss of information. Salt \& Pepper is a form of noise which occurs randomly in an image as white and black pixels. Traditionally, median filter is used to remove this kind of noise but it introduces blurring in image which causes loss of small details. In this paper Fuzzy Logic based Adaptive Median Filter (FL-AMF) using MATLAB ${ }^{\circledR}$ is proposed which removes the noise effectively and also preserves small details. By introducing various densities of noise, performance of both filters are compared using PSNR \& it is found that FLAMF gives better results.
\end{abstract}

\section{Keywords}

FL-AMF, Fuzzy Inference System, PSNR, Membership set.

\section{INTRODUCTION}

An image contains a lot of information. Noise can degrade the image which causes loss of information. The main sources of noise in digital images arise during image acquisition and/or transmission. The performance of imaging sensor and transmission depends on various factors such as environmental conditions during acquisition and atmospheric condition during transmission [1].

Noise restoration is a vital part of digital image processing. To remove noise one must be wholly aware about the type of noise causing degradation in the image. In this paper, Salt and Pepper noise is used to degrade the image. For de-noising or restoration this kind of noise, conventionally median filter is used. Median filter changes value of every pixel in an image by median value of selected neighborhood of dimension ( $\mathrm{m}$, n). Salt and Pepper noise is also known as on-off noise, it is of two types Bi-polar and Uni-polar. It randomly introduces white and/or black pixels in an image. White pixel corresponds to maximum value and black pixel corresponds to minimum value of an image [1]. Instead of processing every pixel of an image, proposed FL-AMF first detects the noisy pixels and then produces a decision based on human reasoning about the actual value of that pixel. Simultaneously, median filter is also used for the same neighborhood and decision value is computed. By this method two values are obtained which can be used as replacement for noisy pixel. But for better results, again Fuzzy method is used to compute one value from these two calculated values. This final value is substituted for noisy pixel.

Following this introduction a brief background of the work based on the fuzzy logic and data used is presented in section 2. The proposed fuzzy based algorithm is described in section 3. Explanation of result is given in section 5. Section 6 concludes the work done and suggests future directions for further improvements.

\section{BACKGROUND}

In recent years, the number and variety of applications of fuzzy logic have increased significantly. The applications range from consumer products such as cameras, camcorders, washing machines, and microwave ovens to industrial process control, medical instrumentation, decision-support systems, and portfolio selection [1]. In 1965, Zadeh proposed a complete theory of fuzzy sets to represent and manipulate illdefined concepts and according to Zadeh " in contrast to traditional hard computing, soft computing exploits the tolerance for imprecision, uncertainty, and partial truth to achieve tractability, robustness, low solution-cost, and better rapport with reality" [3].Fuzzy Inference Systems (FIS) are one of the better known applications of fuzzy set theory. They can be seen as a mapping between sets of fuzzy sets. Let X, Y be non-empty sets and $\mathrm{f}: \mathrm{X} \rightarrow \mathrm{Y}$ be any continuous function. Let $F(X), F(Y)$ denote the set of all fuzzy sets defined on $X$, $\mathrm{Y}$, respectively, i.e., $\mathrm{F}(\mathrm{X})=\{\mathrm{A} \mid \mathrm{A}: \mathrm{X} \rightarrow[0,1]\}$. Let an input $\mathrm{X}$ $\in X$ be given to the fuzzy inference system. With the help of a fuzzy if-then rule base and fuzzy logic operations on $[0,1]$, the inference module determines a corresponding output $y \in$ $Y$. Thus, an FIS approximates $f$ by a fuzzy function $f^{\wedge}: F(X)$ $\rightarrow \mathrm{F}(\mathrm{Y})$ [6]. The inference mechanism in an FIS itself can differ in their strategy of combining rules, choice of fuzzy logic operations, etc., and hence lead to various established types of FIS, viz., TS-fuzzy systems, Mamdani, Relational FIS, etc. A fuzzy inference system can be completely and uniquely characterized by the following 6-tuple [6]:

$\mathrm{F}=(\mathrm{X}, \mathrm{Y}, \mathrm{Ai}, \mathrm{Bi}, \mathrm{R}, \mathrm{O})$, where

- X, Y are the input and output domains;

- Ai, Bi are the fuzzy sets partitioning X, Y;

- $\mathrm{R}$ is a set of $\mathrm{n}$ Single-Input Single-Output fuzzy rules of the form $(\mathrm{i}=1 \ldots \mathrm{n})$ : $\mathrm{Ri}$ : If ${ }^{\sim} \mathrm{x}$ is $\mathrm{Ai}$ then $\mathrm{y}^{\sim}$ is $\mathrm{Bi}$;

- $\mathrm{O}$ is the set of fuzzy logic operations used in the inference.

The probability density function (PDF) Salt \& Pepper (Impulse) noise can be defined as follows:

$p(z)=\left\{\begin{array}{c}P a \text { for } z=a \\ P b \text { for } z=b \\ 0 \text { otherwise }\end{array}\right.$

If $\mathrm{b}>\mathrm{a}$, intensity $\mathrm{b}$ will appear as light dot and vice-versa. $\mathrm{Pa}$ and $\mathrm{Pb}$ define the probability of $\mathrm{a}$ and $\mathrm{b}$ respectively. If either $\mathrm{Pa}$ or $\mathrm{Pb}$ is zero then it is called as the uni-polar noise. For salt 
\& pepper noise, $\mathrm{Pa}$ must be equal to $\mathrm{Pb}$ and is also referred as Data-drop-out and spike noise [1].

PSNR (db) is peak signal to noise ratio and given as follows:

$$
M S E=\frac{\sum_{M, N}\left[I_{1}(m, n)-I_{2}(m, n)\right]^{2}}{M^{*} N} \mid P S N R=10 \log _{10}\left(\frac{R^{2}}{M S E}\right)
$$

$M S E$ is the mean square error. $I$ is the image matrix. $M$ and $N$ are the number of rows and columns in the input images, respectively. $R$ is the maximum fluctuation in the input image data type. For example, if the input image has a doubleprecision floating-point data type, then $R$ is 1 . If it has an 8-bit unsigned integer data type, $R$ is 255 , etc [2].

\section{PROPOSED SCHEME}

Fuzzy inference system used in proposed method is 2-inputs and 1-output of Mamdani type.

$\mu_{\mathrm{c}}(\mathrm{y})=\min _{\mathrm{k}}\left[\min \left[\mu_{\mathrm{A}}(\operatorname{input}(\mathrm{i})), \mu_{\mathrm{B}}(\operatorname{input}(\mathrm{j})),\right]\right]$

$\mathrm{k}=1,2 \ldots \mathrm{r}$.

For Fuzzification of input, Inference method is used in which membership function is defined by means of various shapes. Membership function defines the information contained in the fuzzy set. A fuzzy set $\tilde{A}$ in the universe of discourse $X$ can be defined as, $\tilde{A}=\left\{\left(x, \mu_{\tilde{A}}(x)\right) \mid x \in X\right\}$, where $\mu_{\tilde{A}}($.) is called membership function of $\tilde{\mathrm{A}}$. The membership value ranges in the interval $[0,1]$. Gaussian shaped membership functions are used as they provide smooth transition [2]. After Inference process, Defuzzification (linguistic variables to numbers) of values is required. For Defuzzification, Centroid method is used and defuzzified output $x^{*}$ is defined as:

$$
x^{*}=\left(\int \mu \tilde{N}(x) \cdot x d x\right) /\left(\int \mu \tilde{N}(x) d x\right)
$$

( $\int$ denotes algebraic integration).

In proposed scheme image restoration is performed in spatial domain. Because as already defined, Salt \& Pepper noise can be easily identified by inspecting each pixel value as either 0 or 255 .

Figure 1 represents Algorithm of Fuzzy Logic Based Adaptive Mean Filter (FL-AMF) and is explained in four stages. First Stage corresponds to detection of noisy pixel. Each pixel of image is inspected one by one. Whenever a noisy pixel [0 or 255 ] is detected, a desired neighborhood ( $3 \times 3$ in this paper) is selected and proper sets are formed from these 9 pixel elements to compute the result.

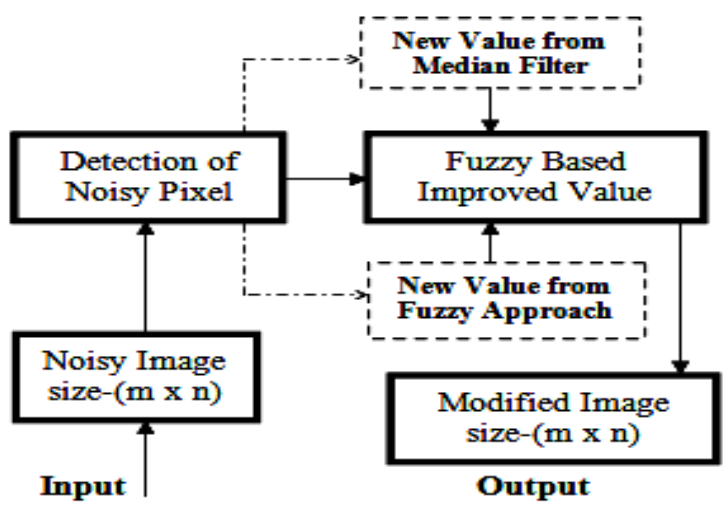

Fig 1: Block Diagram of FL-AMF process

In the selected neighborhood as shown below, four sets of two pixels each are formed. These four sets are; Set1 - [E2, E8], Set2 - [E4, E6], Set3 - [E1, E9] \& Set4 - [E3, E7]. Each set has two pixels and labeled as $\mathrm{P} 1, \mathrm{P} 2$ in further explanations.

\begin{tabular}{|c|c|c|}
\hline El & E2 & E3 \\
N-W & North & N-E \\
\hline E4 & E5 & E6 \\
West & Noisy Pixel & East \\
\hline E7 & E8 & E9 \\
S-W & South & S-E \\
\hline
\end{tabular}

Fig 2: Selected 3x3 Neighborhood
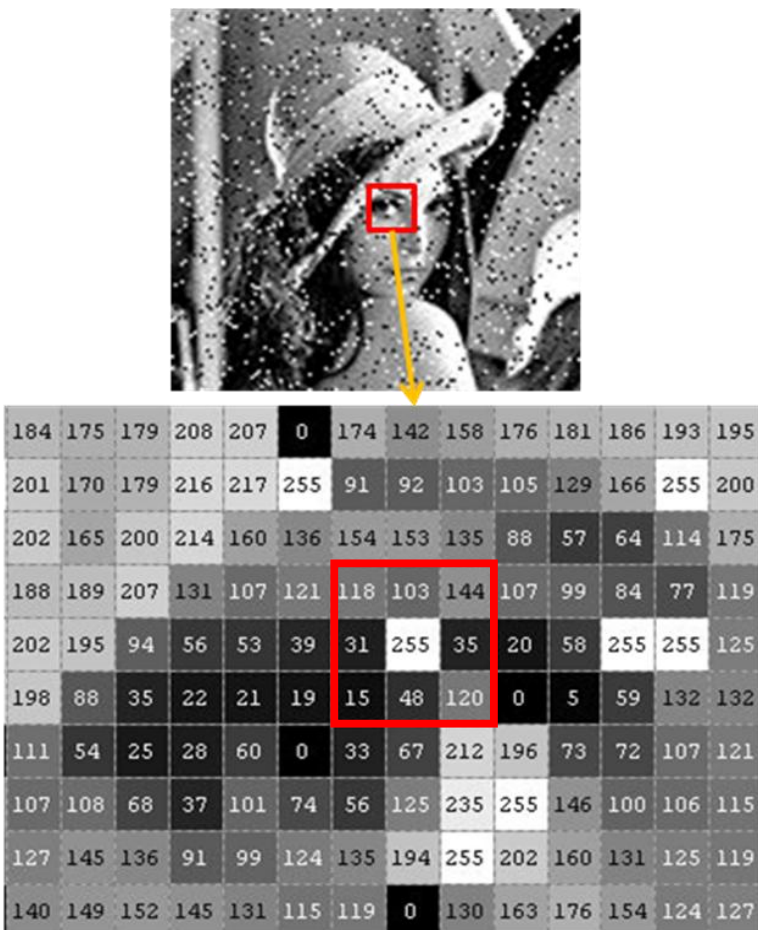

Fig 3: Selected 3x3 Neighborhood from Lena Image

DP1= median [15 313548103120144118 255]

DP1=103 and median filter replaces all the centre pixels with, DP1 value in same way, irrespective of pixel is noisy or not.

FL-AMF filter will process $3 \times 3$ neighborhood only if pixel is noisy (Noisy Pixel is [0 or 255]). Four sets will be formed \& processed as follows:

$\mathrm{s} 1=[103,48] ; \mathrm{s} 2=[31,35] ; \mathrm{s} 3=[118,120] ; \mathrm{s} 4=[144,15] ;$

$\mathrm{NS} 1=[$ fuzzy $(\mathrm{s} 1)$, fuzzy $(\mathrm{s} 2)]=$ fuzzy $[76,37.3]=68.4$

$\mathrm{NS} 2=[$ fuzzy $(\mathrm{s} 3)$, fuzzy $(\mathrm{s} 4)]=$ fuzzy $[25,75.5]=40$

$\mathrm{DP} 2=$ fuzzy $(\mathrm{NS} 1, \mathrm{NS} 2)=45.3$

In Third Stage the two final values obtained, are again processed by FL-AMF to deduce the final value. Fourth Stage 
replaces the value of noisy pixel by new pixel obtained in previous step. This process is repeated until all noisy pixels of image are modified.

$$
\mathrm{f}=\text { fuzzy }(\mathrm{DP} 1, \mathrm{DP} 2)=30
$$

Finally, the noisy pixel will be replaced by the value $f=30$.

FIS used here has 3 sets in membership function (Figure-2) for each of the inputs and 5 sets in membership function for each (Figure-3) of output. Rule base contains total of nine rules as 2 inputs have 3 set each $(3 \times 3)$. Figure- 4 represents the surface viewer for relationship between inputs and output according to rule base.

Fuzzy rule base for the system is deduced by mapping the input values to the output. For example:

\section{If (P1 is Dark) and (P2 is Dark) then (Decision is Dark).}

2. If (P1 is Light) and (P2 is Dark) then (Decision is Avg).

In the similar way all 9 rules are deduced. P1 and P2 define the pixel set from neighborhood and are considered as input to fuzzy system. To compute the complete neighborhood ( $3 \times 3)$ except center pixel which is noisy (obtained in first stage of detection.), firstly four sets are formed from eight pixels. Clubbing of pixels is done with respect to centre pixel as: First set corresponds to N (North) and S (South) pixels,

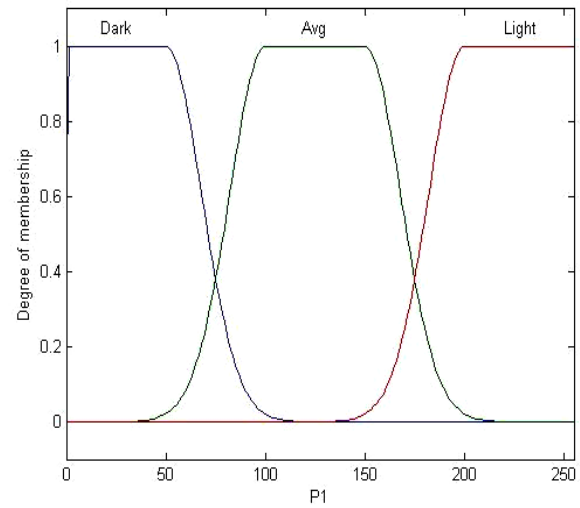

Fig 4: Membership Function for Input

Second set corresponds to W (west) and E (East) pixels Third set corresponds to NE (North-East) and SW (South-West) pixels and Fourth set corresponds to NW (North-West) and SE (South-East) pixels. These 4 sets are fed to FIS one by one and 4 pixels are obtained as the result.

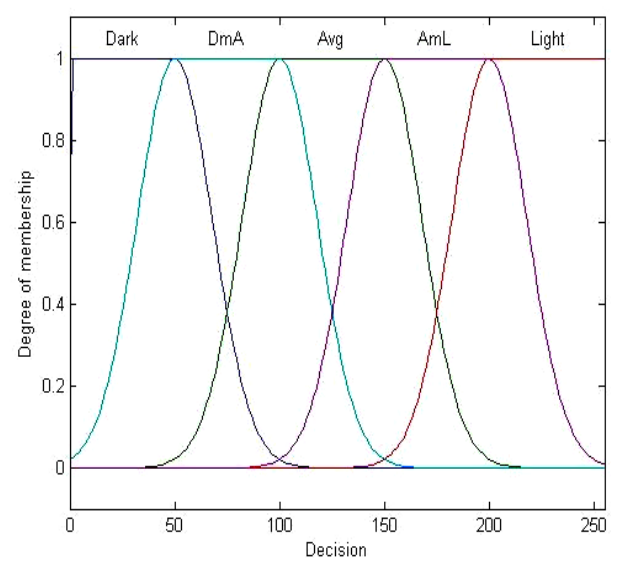

Fig 5: Membership Function for Output

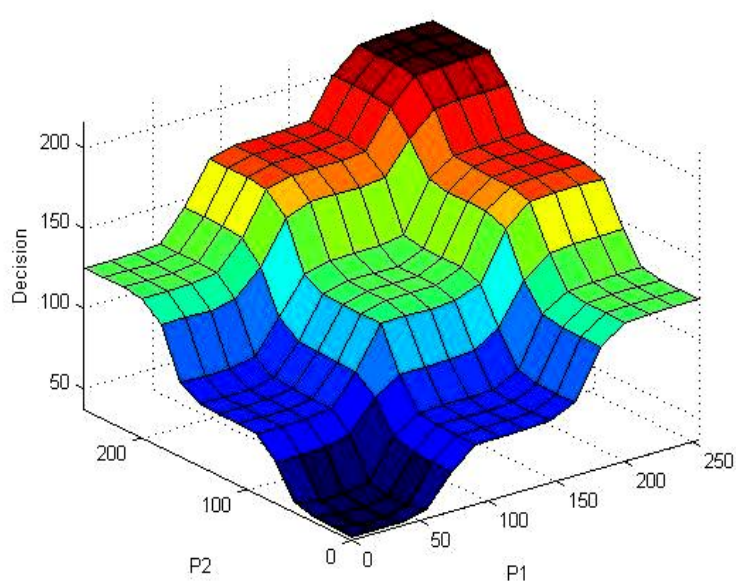

Fig 6: Surface Viewer

Again clubbing of 4 pixels in two sets is done according to weight and fed to FIS, which results in one set of 2 pixels and finally processed by FIS once again into one decision pixel (DP1). The same neighborhood is processed by median filter and resultant pixel is considered as DP2. By this process two values are obtained which can be replaced in central pixel.
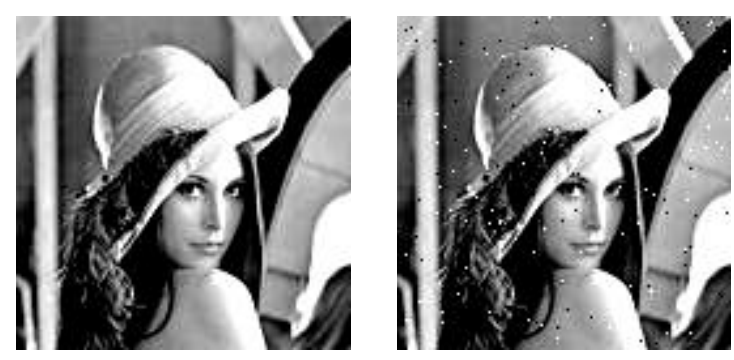

(a)

(b) 


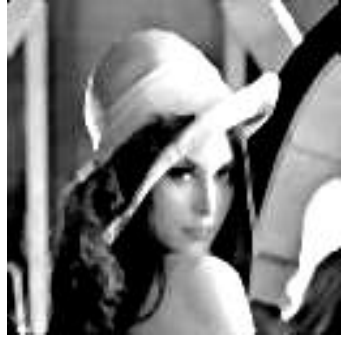

(c)

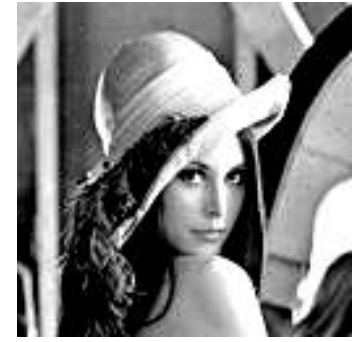

(d)
Fig 7: Results for Lena Image

(a) Original Image, (b) Corrupted Image with $10 \%$ noise, (c) Median Filtered Image, (d) FZ-AMF Filtered Image

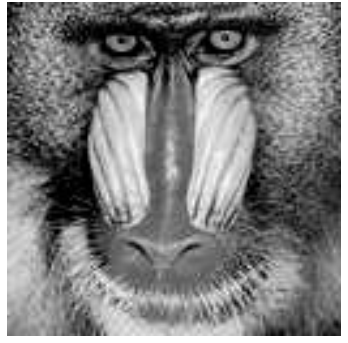

(a)

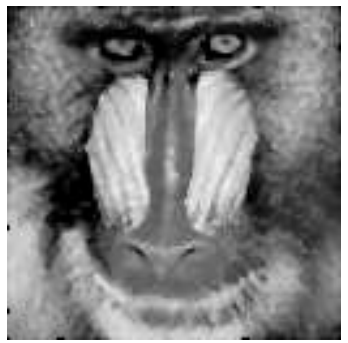

(c)

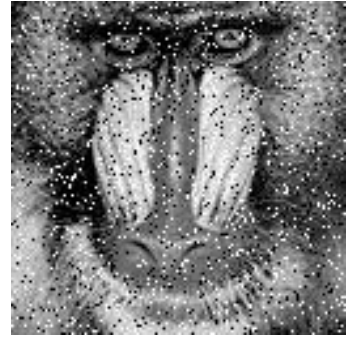

(b)

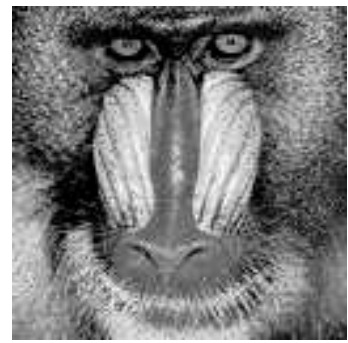

(d)
Fig 8: Results for Mandril Image

(a) Original Image, (b) Corrupted Image with $70 \%$ noise, (c) Median Filtered Image, (d) FZ-AMF Filtered Image

But again to deduce the better value by DP1 and DP2 both values are fed to FIS and final decision value is obtained and used as replacement for noisy or central pixel.

Figure-5 (Lena image) and Figure-6 (Mandril image) represents the final results of proposed approach and traditional median filter. Figure-7 represents pixel profile.

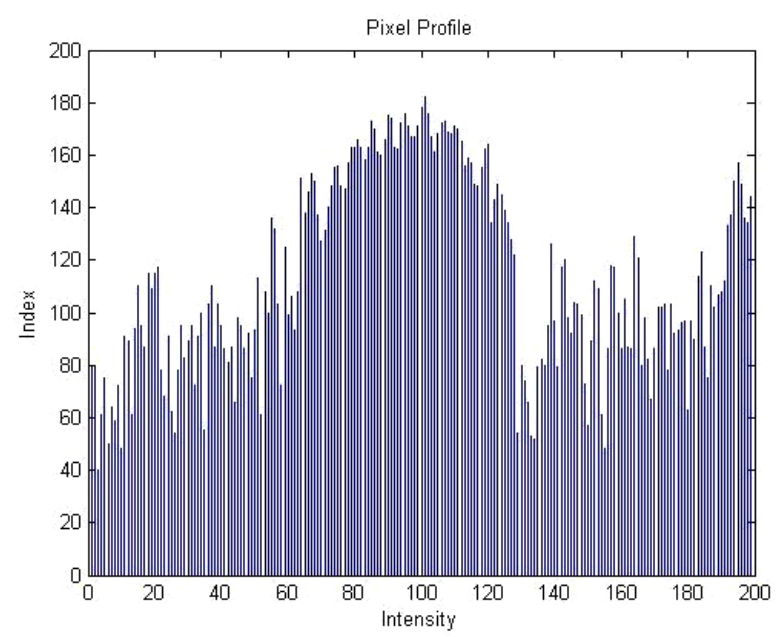

(a)

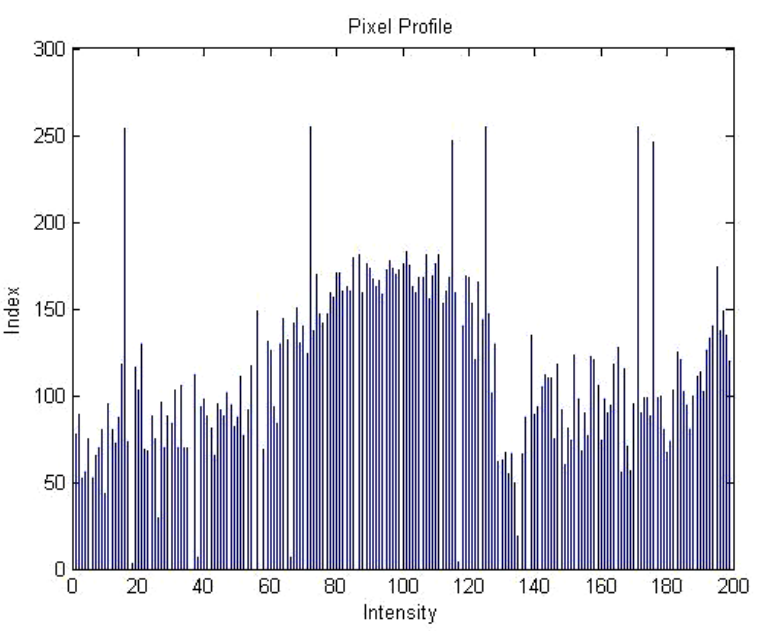

(b)

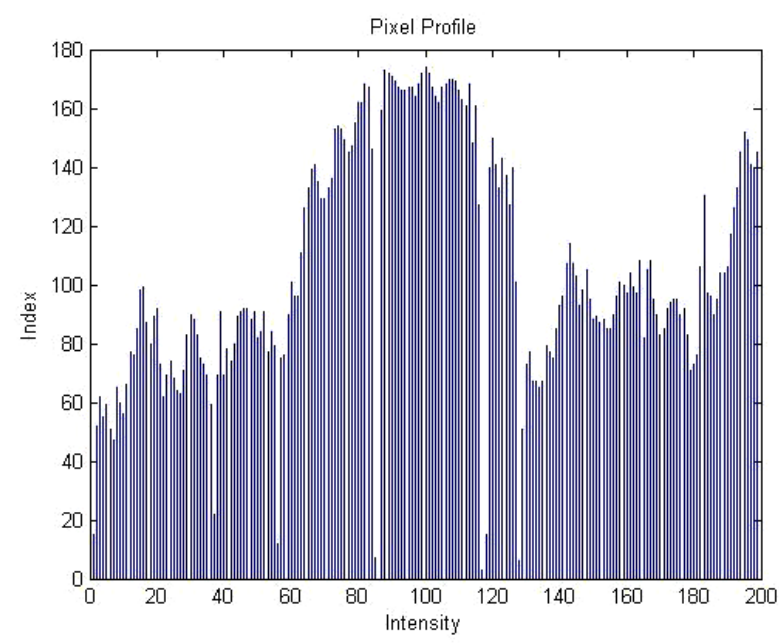

(c) 


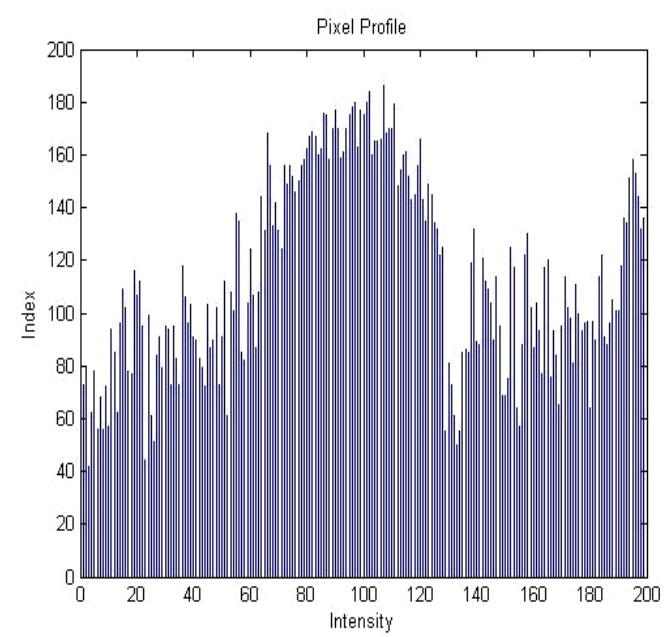

(d)

Fig 9: Pixel Profile for Mandril Image

(a) Original Image, (b) Corrupted Image with $70 \%$ noise, (c) Median Filtered Image, (d) FZ-AMF Filtered Image

\section{RESULTS}

Median Filter \& FL-AMF are used and compared by PSNR value for a number of images and it is found that, FL-AMF performs well. Figure 8 represents PSNR values for Lena image $(512 \times 512)$ for a various range of salt and pepper noise densities. Noise pixels on boundary of image are left almost untreated by median filter.

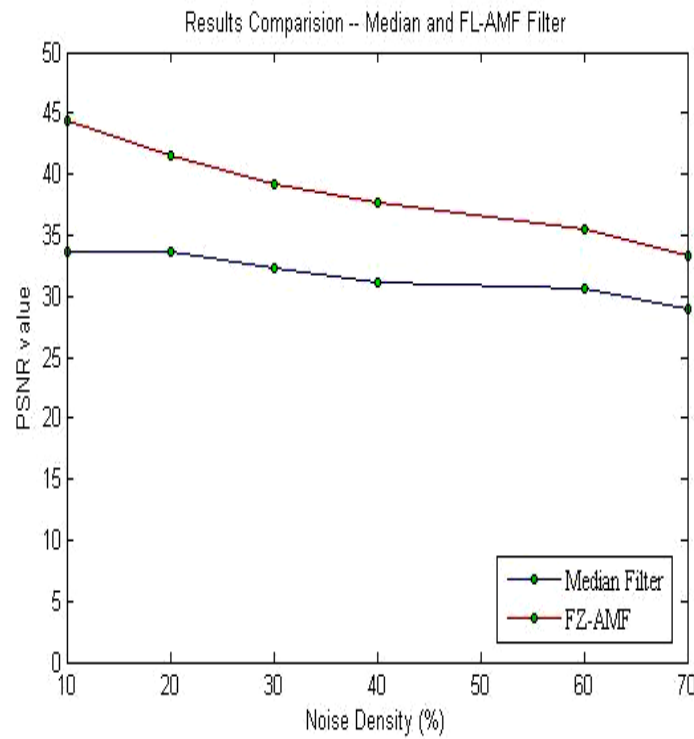

Fig 10: Comparison Results of PSNR - Median and FLAMF Filters

Noise on boundary and preservation of Small details (edge details) are considered effectively by FL-AMF and can be visualized easily in Figure 7 which represents pixel profile for Mandril image.

\section{CONCLUSION}

In this paper proposed scheme performs very well in comparison to median filter for grayscale images. For further research, area of improvisation can be removal of Salt \& Pepper noise in colored images and use of FL-AMF for denoising of other noises. Iterative processing can also be considered for image enhancement purpose.

\section{REFERENCES}

[1] R. C. Gonzalez and R. E. Woods. Digital Image Processing. Pearson, 2011

[2] S.N. Sivanandam, S. N. Deepa Principles of soft computing. John Wiley \& Sons, Inc, 2009.

[3] Fuzzy Logic Tool Box user guide Matlab(R2009b)

[4] A. B. Badiru, J. Y. Cheung, Fuzzy Engineering Expert Systems. John Wiley \& Sons, Inc.

[5] T. J. Ross, Fuzzy Logic with engineering applications. Wiley

[6] B. Jayaram, "Fuzzy Inference System based Contrast Enhancement", EUSFLAT-LFA2011

[7] H. Xu, "An Adaptive Fuzzy Switching filter for images corrupted by impulsive noise" Sixth international conference on Fuzzy systems and Knowledge Discovery, 2009.

[8] Y. Zhou, "Adaptive Fuzzy Median Filter for images corrupted by impulsive noise", Congress on Image and Signal Processing, 2008.

[9] S. Zhang and M.A. Karim, -A new impulse detector for switching median filtersl, IEEE Signal Processing Letters, vol 9, no 11, pp 360-363, Nov 2002.

[10] R. H. Chan, C. Ho, and M. Nikolova," Salt-andPepper Noise Removal by Median-type Noise Detectors and Detail- preserving Regularization," IEEE Transaction on Image Processing, Vol. 14, No. 10, Oct, 2005.

[11] K. M. Singh, "Fuzzy Rule based Median filter for GrayScale Images, "Journal of information Hiding and Multimedia signal processing,vol.2,no.2, April 2011.

[12] H. Kundra," Filter for removal of impulse noise by using fuzzy logic", International Journal of image processing (IJIP), vol.3, issue 3, March 2011.

[13] J. Kaur, "SALT \& PEPPER NOISE REMOVAL USING FUZZY BASED ADAPTIVE FILTER", International Journal of Science, Engineering and Technology Research (IJSETR) Volume 1, Issue 1, July 2012.

[14] Z. F. Deng, Z. P. Yin, and Y. L. Xiong, "High probability impulse noise-removing algorithm based on mathematical morphology," IEEE Signal Process. Lett., vol. 14, no. 1, pp. 31-34, Jan. 2007.

[15] Pei-Yin Chen, Chih-Yuan Lien, "An Efficient EdgePreserving Algorithm for Removal of Salt-and-Pepper Noise”, IEEE Signal Processing Letters, vol. 15, 2008. 